\title{
Effects of flavonoids on glycosaminoglycan synthesis: implications for substrate reduction therapy in Sanfilippo disease and other mucopolysaccharidoses
}

\author{
Anna Kloska • Joanna Jakóbkiewicz-Banecka • \\ Magdalena Narajczyk • Zyta Banecka-Majkutewicz • \\ Grzegorz Węgrzyn
}

Received: 30 October 2010 /Accepted: 18 January 2011 /Published online: 9 February 2011

(C) The Author(s) 2011. This article is published with open access at Springerlink.com

\begin{abstract}
Sanfilippo disease (mucopolysaccharidosis type III, MPS III) is a severe metabolic disorder caused by accumulation of heparan sulfate (HS), one of glycosaminoglycans (GAGs), due to a genetic defect resulting in a deficiency of GAG hydrolysis. This disorder is characterized as the most severe neurological form of MPS, revealing rapid deterioration of brain functions. Among therapeutic approaches for MPS III, one of the most promising appears to be the substrate reduction therapy (SRT). Genistein (5, 7-dihydroxy-3- (4-hydroxyphenyl)4H-1-benzopyran-4-one) is an isoflavone that has been used in SRT for MPS III. In this report, we tested effects of other flavonoids (apigenin, daidzein, kaempferol and naringenin) on GAG synthesis. Their cytotoxicity and anti-proliferation features were also tested. We found that daidzein and kaempferol inhibited GAG synthesis significantly. Moreover, these compounds were able to reduce lysosomal storage in MPS IIIA fibroblasts. Interestingly, although genistein is believed to inhibit GAG synthesis by blocking the tyrosine kinase activity of the epidermal growth factor receptor, we found that effects of other
\end{abstract}

A. Kloska · J. Jakóbkiewicz-Banecka · G. Węgrzyn $(\bowtie)$ Department of Molecular Biology, University of Gdańsk, Kładki 24, 80-822 Gdańsk, Poland

e-mail: wegrzyn@biotech.ug.gda.pl

M. Narajczyk

Laboratory of Electron Microscopy, Faculty of Biology,

University of Gdańsk,

Kładki 24, 80-822 Gdańsk, Poland

Z. Banecka-Majkutewicz

Department of Neurology, Medical University of Gdańsk,

Dębinki 7, 80-211 Gdańsk, Poland flavonoids were not due to this mechanism. In fact, combinations of various flavonoids resulted in significantly more effective inhibition of GAG synthesis than the use of any of these compounds alone. These results, together with results published recently by others, suggest that combination of flavonoids can be considered as a method for improvement of efficiency of SRT for MPS III.

Keywords Flavonoids · Mucopolysaccharidoses · Substrate reduction therapy. Glycosaminoglycan synthesis

\section{Introduction}

Mucopolysaccharidoses (MPS) are inherited metabolic disorders, caused by mutations leading to dysfunction of one of enzymes involved in degradation of glycosaminoglycans (GAGs) in lysosomes (Beck 2007a; Neufeld and Muenzer 2001). Depending on the nature of the deficient enzyme, eleven types and subtypes of MPS are already recognized. Partially degraded GAGs accumulate in cells of MPS patients, causing dysfunction of tissues and organs, including the heart, respiratory system, bones, joints and central nervous system (CNS). In MPS types characterized by the storage of heparan sulfate (HS), one of the GAGs, neurological symptoms are among the most severe ones (Neufeld and Muenzer 2001). Although enzyme replacement therapy (ERT) has been developed for various types of MPS, and registered to date for three of them (types I, II and VI), such a treatment is ineffective for neurological symptoms as the enzyme cannot cross the blood-brainbarrier (for reviews, see Beck 2007a, b). The second therapy used in a relatively large fraction of MPS patients, 
bone marrow transplantation, has been shown to be efficient to some extent in MPS type I, but not in MPS III (Sanfilippo disease) (Beck 2007a, b), an MPS type characterized by the most severe CNS dysfunctions among mucopolysaccharidoses (for recent discussion, see Węgrzyn et al. 2010).

Recently, 5, 7-dihydroxy-3- (4-hydroxyphenyl)-4H-1benzopyran-4-one), known also as genistein or 4', 5, 7trihydroxyisoflavone, has been demonstrated to be an inhibitor of GAG synthesis in fibroblasts of patients suffering from various types of mucopolysaccharidoses, including MPS III (Piotrowska et al. 2006). Importantly, a decrease in lysosomal GAG storage was noted after incubation of the cells with genistein (Piotrowska et al. 2006). Subsequent studies indicated that genistein may be effective in treatment of mice suffering from MPS IIIB (one of four subtypes of MPS III) and MPS II, including improvement in CNS in short-term treatment of animals with the latter disease and complete correction of behavior in long-term treatment of animals with the former disease (Friso et al. 2010; Malinowska et al. 2009, 2010). In pilot clinical studies, it was demonstrated that treatment of patients suffering from MPS IIIA and MPS IIIB with a genistein-rich isoflavone extract resulted in statistically important improvement of all tested parameters (when mean values from all patients were compared), including cognitive functions, assessed by using a special psychological test (Piotrowska et al. 2008). The effects of genistein on neurological parameters in MPS animals and humans were assumed to be due to an ability of this isoflavone to cross the blood-brain-barrier, which was demonstrated previously (Tsai 2005).

The mechanism of the genistein-mediated substrate reduction therapy (SRT) was proposed to be due to inhibition of phosphorylation of epidermal growth factor receptor (EGFR) by this isoflavone (Jakobkiewicz-Banecka et al. 2009). This therapeutic approach is promising (Jakobkiewicz-Banecka et al. 2007), however, it is also clear that further studies are necessary to optimize this procedure. For example, Arfi et al. (2010) tested various isoflavones for their activities in correction of GAG lysosomal accumulation in MPS IIIA and MPS VII fibroblasts, and found that some of the tested compounds are as efficient as genistein in decreasing GAG storage in MPS cells, and combination of different compounds from this group may be more efficient than one single flavonoid. Therefore, in this work we tested effects of some other already known flavonoids - not only isoflavones (genistein and daidzein, as studied by Arfi et al. 2010), but also a flavone (apigenin), a flavonol (kaempferol) and a flavanone (naringenin)-on GAG synthesis, considering that these compounds might be potentially useful in optimization of SRT for MPS.

\section{Materials and methods}

Cell lines, culture media, supplements and flavonoid solutions

Skin fibroblasts obtained form MPS IIIA and MPS IIIB patients (Piotrowska et al. 2009) were used in all experiments and Human Dermal Fibroblasts adult cell line (Cascade Biologics, Portland, OR, USA) was used as a healthy control line. Cells were cultured in Dulbecco's modified Eagle's medium (DMEM) supplemented with $10 \%$ fetal bovine serum (FBS) and $1 \times$ Antibiotic and Antimycotic solution (all purchased from Sigma, Germany) and incubated at $37^{\circ} \mathrm{C}$ in humidified $5 \% \mathrm{CO}_{2}$ atmosphere. Minimal Essential Medium without inorganic sulfates (MEM, Joklik's modified, Sigma, Germany) was used in GAG synthesis assay. Phosphate Buffered Saline (PBS), dimethylsulfoxide (DMSO) and dimethylformamide (DMF) were from Sigma (Germany). Naringenin, daidzein, apigenin and kaempferol were purchased from Sigma (Germany) and genistein was synthesized at the Pharmaceutical Research Institute (Warsaw, Poland). Stock solutions of flavonoids were prepared in dimethylformamide (DMF). MTT reagent (3-(4,5dimethylthiazol-2-yl)-2,5-diphenyltetrazolium bromide) was dissolved in RPMI-1,640 medium without phenol red (purchased from Sigma, Germany).

\section{Cytotoxicity and proliferation assay}

Cell viability was assessed with MTT assay. In brief, $6 \times$ $10^{3}$ cells per well (in cytotoxicity assay) or $10^{3}$ cells per well (in proliferation assay) were seed in 96-well plates and incubated overnight and than the growth medium was substituted with fresh medium supplemented with tested compounds at appropriate concentrations or $0.05 \% \mathrm{DMF}$ as a control (in cultures with tested compounds, DMF final concentration was also adjusted to $0.05 \%$ ). Following incubation for $24 \mathrm{~h}$ (to test cytotoxicity) or for 7-days (to test cell proliferation), medium was substituted with MTT (3-(4,5-dimethylthiazol-2-yl)-2,5-diphenyltetrazolium bromide) solution ( $1 \mathrm{mg} / \mathrm{ml}$ in RPMI-1640 medium) and after 2-h incubation at $37^{\circ} \mathrm{C}$ the formazan product was dissolved in DMSO and absorbance was read at $550 \mathrm{~nm}$. $\mathrm{LC}_{50}$ value (in cytotoxicity assay) or $\mathrm{IC}_{50}$ value (in proliferation assay) was determined for each compound in contrast to nontreated cultures (incubated with DMF only).

Measurement of kinetics of GAG synthesis

Kinetics of GAG synthesis was analyzed by measurement of ${ }^{35} \mathrm{~S}$ incorporation into proteoglycans according to previously 
described procedure (Piotrowska et al. 2006) with minor modifications. In brief, cells in a number of $2 \times 10^{4}$ per well were plated in 48-well plates and incubated overnight. Growth medium was substituted with medium supplemented with tested flavonoids at appropriate concentrations or $0.05 \%$ $\mathrm{DMF}$ as a control. Cells were incubated for 48 -hours and next labeled with $20 \mu \mathrm{Ci} / \mathrm{ml}$ of $\mathrm{H}_{2}\left[{ }^{35} \mathrm{~S}^{\mathrm{O}} \mathrm{O}_{4}\right.$ (Hartmann Analytic $\mathrm{GmbH}$, Germany) in a growth medium without inorganic sulfate (MEM, Joklik's modified) mixed with standard DMEM medium (1:1) suplemented with FBS and tested compounds. Cells were grown for another $24 \mathrm{~h}$, washed six times with PBS, digested with $0.03 \%$ papain (prepared in $100 \mathrm{mM}$ sodium acetate with $5 \mathrm{mM}$ L-cysteine, $\mathrm{pH}$ 7.0) (Merck, Germany) and ${ }^{35} \mathrm{~S}$ incorporation was measured in a scintillation counter. DNA concentration in papain digested samples was determined using Quant-iTTM PicoGreen ${ }^{\circledR}$ dsDNA Reagent [Molecular Probes, Inc., Eugene, OR, USA] according to the manufacturer's protocol. Incorporation of ${ }^{35} \mathrm{~S}$ was calculated per DNA amount.

\section{Electron microscopic studies}

Cells were grown in 12-well plate in DMEM supplemented with appropriate concentrations of tested compounds for 6 days, washed three times with PBS, fixed with $2.5 \%$ glutaraldehyde, next fixed in $1 \%$ osmium tetroxide and $1 \%$ potassium hexacyanoferrate(III). Following ethanol dehydration cells were embedded in Epon 812 resin (Fluka, Germany), cut on the utlramicrotom PowerTome XL and contrasted in lead acetate and uranyl acetate. Transmission electron microscopy studies were performed using a Philips CM100 microscope and lysosome structure number was determined.

Assessment of tyrosine kinase activity of EGF receptor

Tyrosine kinase activity of EGF receptor was assessed using commercially available Cell-Based ELISA, Human Phospho-EGF R (Y1068) Immunoassay (R\&D Systems Inc., Minneapolis, MN, USA). Cells were plated in a number of $10^{4}$ cells per well of 96-well plate and incubated overnight. Growth medium was substituted with medium supplemented with flavonoids or a potent tyrosine kinase inhibitor PD168393 (Calbiochem, La Jolla, CA, USA) in appropriate concentrations. Following 2-h incubation, Epidermal Growth Factor (BD Biosciences, Bedford, MA, USA) at the concentration of $100 \mathrm{ng} / \mathrm{ml}$ was added to each well for $15 \mathrm{~min}$. in order to induce EGF receptor autophosphorylation. Next, growth medium was removed, cells were fixed with $4 \%$ formaldehyde in PBS for $20 \mathrm{~min}$. and following steps were performed according to the protocol provided by the manufacturer of the ELISA assay.

\section{Statistical analysis}

To test for the effects of different concentrations of flavonoids on the number of lysosomal structures one-way ANOVA was performed. Tukey's multiple comparisions test was used as a post-hoc comparator. This test, and the t-Student two-tailed test were used to assess effects of flavonoids and their mixtures on GAG synthesis efficiency and on the kinase activity of EGFR. All tests were performed using Statistica 8.0 [StatSoft, Poland] software with significance indicated where $p$ values were less than 0.05 .

\section{Results}

Cytotoxic and antiproliferative activities of flavonoids

We have tested different natural flavonoids for their activities as inhibitors of GAG synthesis, and thus, for their potential use in SRT for MPS. Apart from genistein, a previously investigated isoflavone, four compounds were tested: apigenin (a flavone), daidzein (an isoflavone), kaempferol (a flavonol) and naringenin (a flavanone) (see Fig. 1 for their formulas).

Potential medicines should reveal low cytotoxicity and acceptable antiproliferative activity, thus, we have tested
Fig. 1 Structural formulas of natural flavonoids used in this work: apigenin (a), daidzein (b), kaempferol (c), naringenin (d) and genistein (e)<smiles></smiles><smiles></smiles><smiles>O=C1CC(c2ccc(O)cc2)Oc2cc(O)cc(O)c21</smiles>

D<smiles>O=c1c(-c2ccc(O)cc2)coc2cc(O)cc(O)c12</smiles> 
these features of the tested flavonoids. We found low cytotoxicity, assessed as $\mathrm{LC}_{50}$, of all tested compounds (Table 1). The $\mathrm{LC}_{50}$ value was $>100 \mu \mathrm{M}$ for each tested compound, and estimation of exact values of this parameter was not possible due to low solubility of flavonoids in water (and thus in the cell culture medium). Moreover, all compounds had the $\mathrm{IC}_{50}$ value higher than that of genistein in the antiproliferative activity assay (Table 1). Since genistein had been shown previously to be a relatively safe compound for treatment of MPS in both animals and human patients (Klein and King 2007; Malinowska et al. 2009; Piotrowska et al. 2008), we suggest that safety features of the tested flavonoids maybe acceptable at this stage of the study, though more detailed experiments, including tests on animals, are necessary to prove their safety for organisms.

\section{Effects of flavonoids on GAG synthesis}

Effects of flavonoids on GAG synthesis were tested in cultures of fibroblasts. Various concentrations of flavonoids were added to cell cultures and synthesis of GAGs was measured by estimation of the amount of incorporated precursor, $\left[{ }^{35} \mathrm{~S}\right]$ sulfate. This method was chosen as in mammalian cells the incorporation of ${ }^{35} \mathrm{~S}$ from radioactive $\mathrm{Na}_{2} \mathrm{SO}_{4}$ into macromolecules occurs almost exclusively into GAGs (Kato et al. 1995). An inhibition of GAG synthesis was found in the presence of all tested compounds, though the most pronounced impairment of production of GAGs was observed in the presence of kaempferol, and statistically significant differences relative to untreated cells were observed only for kaempferol, daidzein and genistein (Fig. 2). Due to low solubility of apigenin and genistein in water, these compounds could not be tested at the concentration of $100 \mu \mathrm{M}$.

Table 1 Cytotoxic and antiproliferative activity of natural flavonoids. Cytotoxicity is expressed as $\mathrm{LC}_{50}$ index value, i.e. concentration of the tested drug $[\mu \mathrm{M}]$ that is lethal to $50 \%$ of cells in a culture exposed to the drug for $24 \mathrm{~h}$. Exact $\mathrm{LC}_{50}$ values could not be determined due to low solubility of flavonoids, which prevented achievement of their concentrations in cell cultures high enough to measure this parameter precisely. Antiproliferative activity is expressed as $\mathrm{IC}_{50}$ index value, i.e. concentration of the tested drug $[\mu \mathrm{M}]$ that causes $50 \%$ inhibition of cell proliferation in a culture exposed to the drug for 7 days

\begin{tabular}{lcc}
\hline Flavonoid & $\mathrm{LC}_{50}[\mu \mathrm{M}]$ & $\mathrm{IC}_{50}[\mu \mathrm{M}]$ \\
\hline Apigenin & $>100$ & 27.4 \\
Daidzein & $>100$ & 59.8 \\
Kaempferol & $>100$ & 37.9 \\
Naringenin & $>100$ & $>100$ \\
Genistein & $>100$ & 16.9 \\
\hline
\end{tabular}

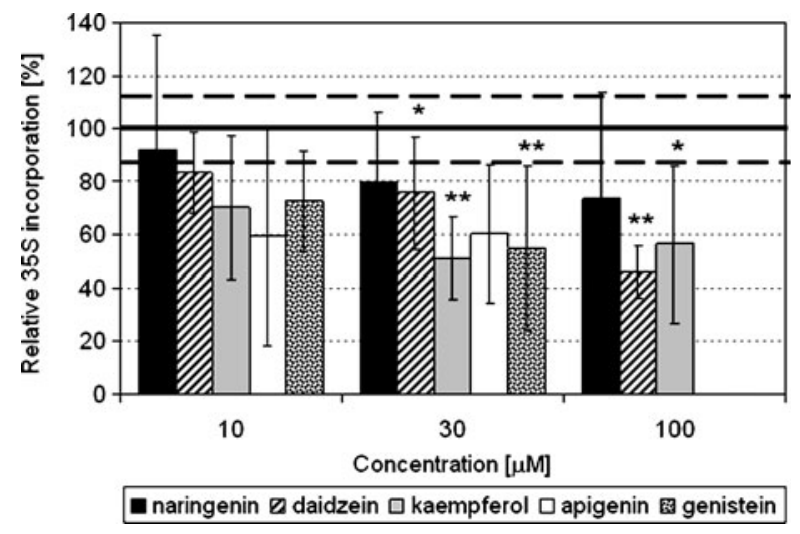

Fig. 2 Dose-dependent effects of natural flavonoids on kinetics of glycosaminoglycan synthesis in fibroblasts presented as relative ${ }^{35} \mathrm{~S}$ incorporation into GAGs after 3-day exposure to different concentrations of flavonoids $[\mu \mathrm{M}]$. Labeling was conducted for $24 \mathrm{~h}$ with $20 \mu \mathrm{Ci} / \mathrm{ml} \mathrm{H}_{2}\left[{ }^{35} \mathrm{~S}_{\mathrm{O}} \mathrm{O}_{4}\right.$. Radioactivity of incorporated ${ }^{35} \mathrm{~S}$ was measured in a scintillation counter, calculated per DNA amount [dpm/ng DNA], and expressed as the percentage of control. 100\% (bold solid line, with SD indicated by bold dashed lines) corresponds to the relative ${ }^{35} \mathrm{~S}$ incorporation into control cells (from the cell culture treated with $0.05 \%$ dimethylformamide). The results presented are average values obtained for five different cell lines with bars indicating standard deviation. Statistical analysis was performed by using the Tukey posthoc test. Values of $p<0.05(*)$ or $p<0.01(* *)$ are indicated

We have also tested effects of combinations of various flavonoids at low concentration $(10 \mu \mathrm{M})$ on GAG synthesis. Interestingly, such combinations resulted in more effective inhibition of GAG production relative not only to particular compounds used at the same concentration alone (Fig. 3), but also to particular compounds used at the concentration as high as the sum of concentrations of all tested flavonoids (compare Figs. 2 and 3). Therefore,

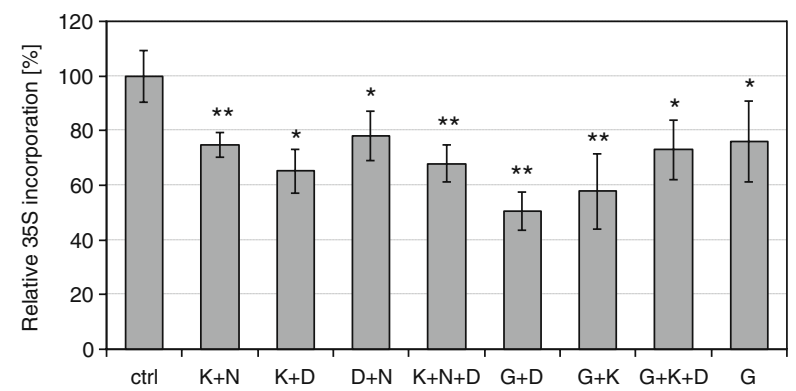

Fig. 3 Effects of mixtures of natural flavonoids (K-kaempferol, $\mathrm{N}-$ naringenin, $\mathrm{D}$ - daidzein, G-genistein, at $10 \mu \mathrm{M}$ concentration each) on kinetics of glycosaminoglycan synthesis in fibroblasts. Relative ${ }^{35} \mathrm{~S}$ incorporation into GAGs after 3-day exposure to mixtures of various flavonoids is presented. Labeling was conducted for $24 \mathrm{~h}$ with $20 \mu \mathrm{Ci} / \mathrm{ml}$ $\mathrm{H}_{2}\left[{ }^{35} \mathrm{~S}_{\mathrm{O}} \mathrm{O}_{4}\right.$. Radioactivity of incorporated ${ }^{35} \mathrm{~S}$ was measured in a scintillation counter, calculated per DNA amount [dpm/ng DNA], and expressed as the percentage of control $(\mathrm{ctrl}=$ cell culture treated with $0.05 \%$ dimethylformamide). The results presented are average values obtained for three different cell lines with bars indicating standard deviation. Statistical analysis was performed by using the $t$-Student twotailed test. Values of $p<0.05(*)$ or $p<0.01(* *)$ are indicated 
additive effects of various flavonoids on impairment of GAG synthesis can be assumed.

Decreased lysosomal storage in the presence of flavonoids

In the treatment of MPS patients, an actual level of lysosomal storage rather than kinetics of GAG synthesis is crucial. Therefore, we have tested effects of flavonoids on changes in lysosomal storage in MPS IIIA fibroblasts. To assess the storage, we used electron microscopic techniques, as described previously (Jakobkiewicz-Banecka et al. 2009; Piotrowska et al. 2006). Examples of abnormal (stored) structures, occurring in MPS IIIA cells, are presented in Fig. 4.

The fibroblasts were cultured in the absence and presence of various flavonoids, and the abnormal structures were observed under electron microscope and counted. We
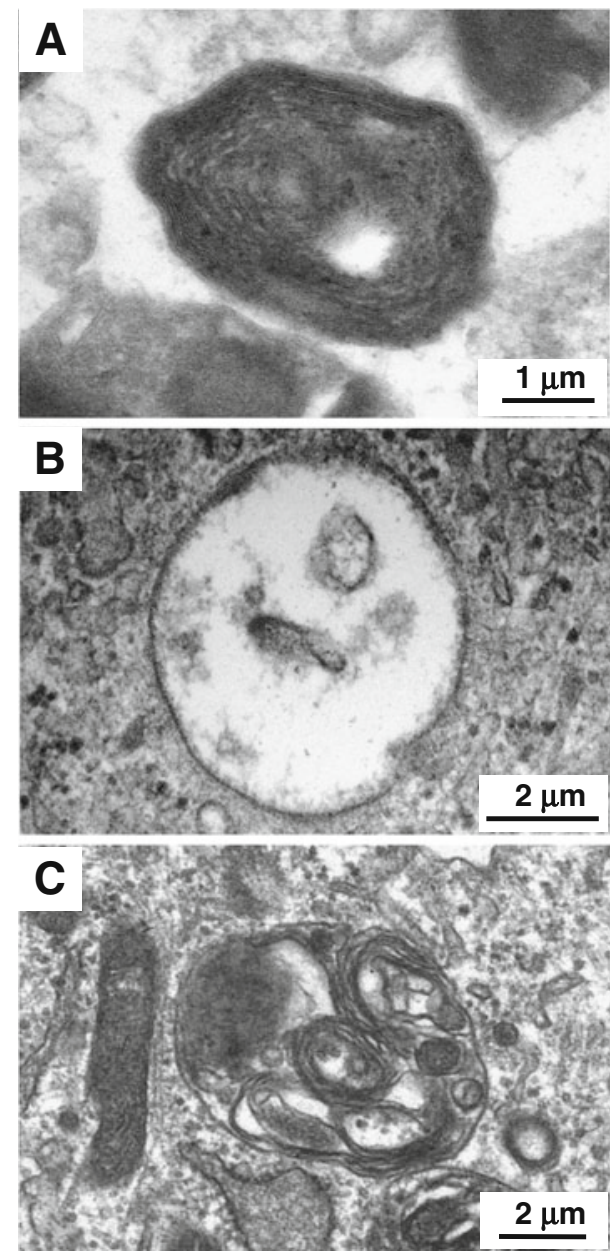

Fig. 4 Different lysosomal structures observed in fibroblasts of the MPS IIIA patient. Electron microphotographs present: lysosome of lamellar and electron-dense structures (a), lysosome of amorphous, flocculent and electron-lucent structures (b), and complex lysosomal structure (autophagolysosome) with storage material of different electron density (c) observed a significant decrease in the number of various abnormal structures in MPS IIIA cells after treatment of the cultures with apigenin, daidzein, kaempferol and naringenin (Table 2). Therefore, we conclude that all tested compounds are effective in elimination of lysosomal storage from MPS cells, despite the fact that only some of these flavonoids (daidzein and kaempferol) caused statistically significant inhibition of GAG synthesis, while the levels of the inhibition by apigenin and naringenin did not reach statistical significance (compare Table 2 and Fig. 2).

Effects of flavonoids on the phosphorylation of epidermal growth factor receptor

Results of previous studies suggested that genistein impairs GAG synthesis due to its inhibitory effect on the kinase activity of the epidermal growth factor receptor (EGFR). Thus, it was proposed that in the presence of genistein the EGFR is underphosphorylated, which correlates with impaired expression of genes coding for enzymes required for GAG synthesis (Jakobkiewicz-Banecka et al. 2009).

To test whether tested flavonoids impair GAG synthesis by the same mechanism as that proposed for genistein, we have measured efficiency of phosphorylation of EGFR in the presence and absence of investigated flavonoids. However, we found that contrary to genistein and a synthetic inhibitor of the tyrosine kinase-PD168393 (used as an additional positive control), none of the tested flavonoids revealed statistically significant inhibiton of EGFR phosphorylation (Fig. 5). Therefore, it appears that these compounds impair GAG synthesis by the meachanism different from that identified for genistein.

\section{Discussion}

Substrate reduction therapy has recently been shown to be a promising strategy for treatment of neuronopathic forms of mucopolysaccharidoses and other lysosomal storage diseases (Jakobkiewicz-Banecka et al. 2007). This therapeutical strategy is based on the use of relatively small molecules, including drugs that may cross the brainblood-barrier and act in the central nervous system. Studies on MPS II and MPS III, the MPS types in which the neurological symptoms are severe or extremely severe, respectively, indicated that genistein, a natural isoflavone, can be effective in treatment of some symptoms, including behavior and cognitive abilities, in both mouse models and human patients (Friso et al. 2010; Malinowska et al. 2009, 2010; Piotrowska et al. 2008). Nevertheless, efficiency of this treatment was not high enough to cure all symptoms in patients, therefore, searching for possibilities to improve SRT for MPS is desirable. 
Table 2 Quantitative analysis of lysosomal structures in MPS IIIA fibroblasts exposed to different concentrations of flavonoids for 6 days. Mean numbers of lysosomal structures per $100 \mu \mathrm{m}^{2}$ of cell cross-section with standard deviation are presented

\begin{tabular}{|c|c|c|c|c|}
\hline \multirow[t]{2}{*}{ Flavonoid $(\mu \mathrm{M})$} & \multicolumn{4}{|c|}{ Number of lysosomal structures per $100 \mu \mathrm{m}^{2}$ of cell cross-section $\pm \mathrm{SD}$} \\
\hline & Lamellar & Complexed (autophago-lysosomes) & Amorphous & Total number \\
\hline Control (DMF) & $0.40 \pm 0.17$ & $0.35 \pm 0.35$ & $0.38 \pm 0.27$ & $1.13 \pm 0.50$ \\
\hline Apigenin (30) & $0.22 \pm 0.18 * \#$ & $0.15 \pm 0.10^{*}$ & $0.14 \pm 0.13 *$ & $0.51 \pm 0.24 *$ \\
\hline Apigenin (100) & $0.08 \pm 0.06^{*} \#$ & $0.20 \pm 0.10$ & $0.13 \pm 0.18^{*}$ & $0.41 \pm 0.18^{*}$ \\
\hline Daidzein (30) & $0.13 \pm 0.12 *$ & $0.12 \pm 0.10^{*}$ & $0.15 \pm 0.11^{*}$ & $0.40 \pm 0.19^{*}$ \\
\hline Daidzein (100) & $0.22 \pm 0.13^{*}$ & $0.32 \pm 0.15$ & $0.16 \pm 0.09 *$ & $0.70 \pm 0.24 *$ \\
\hline Kaempferol (30) & $0.21 \pm 0.12^{*}$ & $0.36 \pm 0.10$ & $0.07 \pm 0.05^{*}$ & $0.63 \pm 0.15^{*}$ \\
\hline Kaempferol (100) & $0.27 \pm 0.26$ & $0.32 \pm 0.13$ & $0.09 \pm 0.06^{*}$ & $0.68 \pm 0.27^{*}$ \\
\hline Naringenin (30) & $0.29 \pm 0.15$ & $0.19 \pm 0.10$ & $0.39 \pm 0.22 \#$ & $0.87 \pm 0.40$ \\
\hline Naringenin (100) & $0.21 \pm 0.17^{*}$ & $0.16 \pm 0.14^{*}$ & $0.19 \pm 0.11 \#$ & $0.56 \pm 0.23 *$ \\
\hline
\end{tabular}

Statistically significant differences (Tukey's post-hoc test; $p<0.05$ ) between control (untreated MPS IIIA cells) and treated cells are indicated with asterisks and significant differences (Tukey's post-hoc test; $p<0.05$ ) between two concentrations of the tested compound are indicated with hash marks

One possibility to achieve higher efficiency of inhibition of GAG synthesis could be the use of the siRNA technology to silence genes responsible for expression of enzymes involved in GAG production, as proposed recently (Dziedzic et al. 2009; Kaidonis et al. 2009). However, the current procedures of delivery of siRNA to central nervous system are not efficient enough to ensure effective treatment.

Another option would be to use different compounds that can inhibit GAG synthesis. Roberts et al. $(2006,2007)$ demonstrated that rhodamine $\mathrm{B}$ is an effective inhibitor of GAG production in vitro and in vivo (in the MPS IIIA mouse model). However, the toxicity profile of rhodamine
B appears to be not acceptable for its use as a drug for humans (Roberts et al. 2006; Roberts et al. 2007). Although recent studies suggest that a long-term treatment with low doses of rhodamine B is safe for mice (Roberts et al. 2010), it is not clear whether the safety profile is the same for humans and whether treatment with such low doses may be effective in alleviation of MPS symptoms. Therefore, we search for other compounds that might be effective in treatment of MPS.

Flavonoids, as compounds relative to genistein that has been demonstrated previously to be an inhibitor of GAG synthesis (Piotrowska et al. 2006), are natural candidates for drugs able to manage neurological symptoms of MPS.

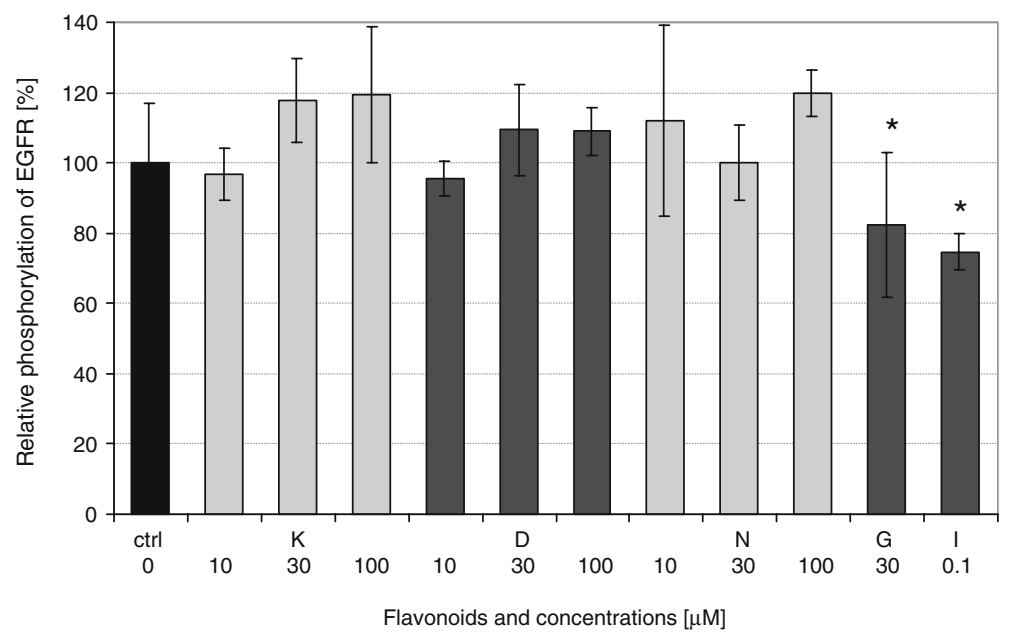

Fig. 5 Effects of flavonoids (at different concentrations) on tyrosine kinase activity of EGF receptor (EGFR). The results of phosphoEGFR fluorescence were normalized to the total EGFR fluorescence and presented as the percentage of the control cells (incubated without flavonoids). Abbreviations are as follows: $\mathrm{K}$-kaempferol, $\mathrm{D}-$ daidzein, $\mathrm{N}$-naringenin, $\mathrm{G}$-genistein, I-a potent EGFR tyrosine kinase inhibitor, PD168390 (which was used as an additional positive control). The results presented are average values obtained from two different experiments with bars indicating standard deviation. Statistically significant differences $(p<0.05)$ are marked by asterisk 
Therefore, we have tested effects of four different flavonoids in assays for GAG synthesis, lysosomal structures and phosphorylation of EGFR. In addition, their cytotoxicity and proliferation inhibition activity were tested.

Our studies revealed that apigenin (a flavone), daidzein (an isoflavone), kaempferol (a flavonol) and naringenin (a flavanone) caused a decrease in the efficiency of GAG synthesis, though only daidzein and kaempferol caused statistically significant differences relative to untreated cells. Nevertheless, in the presence of all these compounds, lysosomal storage in MPS IIIA fibroblasts was significantly decreased. Since all the tested flavonoids, when used at moderate (up to 30 or $100 \mu \mathrm{M}$, depending on the solubility of the compound) concentrations, revealed acceptable profiles in cytotoxicity and proliferation inhibition assays, either in MPS-derived cells (this report) or normal cells (Matsuo et al. 2005; Li et al. 2008; Kwack et al. 2009), it appears that they can be considered as potential drugs. Obviously, further tests, including those with animal models, are necessary to prove their safety for organisms.

Interesting results were obtained when combinations of various flavonoids at their low concentration $(10 \mu \mathrm{M})$ were tested. Many such combinations appeared to be more effective in GAG synthesis inhibition than particular compounds tested separately, even when total flavonoid concentrations were the same. A report by Arfi et al. (2010) has been published recently, in which various isoflavones (daidzein, glycitein, formononetin, prunetin and biochanin) were tested for their activities in correction of GAG lysosomal accumulation in MPS IIIA and MPS VII fibroblasts. Although different methods of estimation of efficiency of these compounds in regulation of GAG metabolism were used by us (this report) and by Arfi et al. (2010), similar conclusions can be drawn from both studies. Namely, some flavonoids (e.g. kaempferol and various isoflavones) are as efficient as genistein in decreasing GAG storage in MPS cells, and combination of different compounds from this group may be more efficient than one single flavonoid. The latter conclusion appears to be reasonable in the light of our results indicating that flavonoids tested in this work (daidzein, kaempferol and naringenin) inhibit GAG synthesis by a mechanism different from that demonstrated for genistein. Namely, contrary to genistein, other flavonoids were not effective in inhibition of EGFR phosphorylation. Although the exact mechanism(s) of their action as GAG synthesis inhibitors remain(s) to be elucidated, differences in means by which various flavonoids impair GAG production may explain their synergistic effects when they were used in mixtures.

Knowing the mechanism of genistein-mediated inhibition of GAG synthesis, which likely depends on inhibition of EGFR autophosphorylation (Jakobkiewicz-Banecka et al. 2009), one might propose that other EGFR tyrosine kinase inhibitors can be considered as putative drugs in MPS treatment. However, there are serious problems with a potential use of such inhibitors in therapies for children suffering from MPS. First, synthetic compounds influencing activities of tyrosine kinases are generally strong inhibitors (Sherman 2009; Takeuchi and Ito 2010), while GAGs are compounds necessary for development and proper functions of many tissues and organs (Bulow and Hobert 2006; Li and Richards 2010), thus, complete or very strong inhibition of their production would not be desired, especially in children. Second, SRT for inherited metabolic diseases, like MPS, must be considered as a long-term therapy, potentially for the whole life. Since there are various toxic and/or adverse effects of synthetic inhibitors of tyrosine kinases reported in literature, including effects on thyroid function and thyroid hormone metabolism (Sherman 2009), diarrhea (Medina and Goodin 2008; Sanford and Scott 2009), nausea (Medina and Goodin 2008), skin toxicity (Li and Perez-Soler 2009), and cardiac toxicity (manifested as congestive heart failure) (de Azambuja et al. 2009; Medina and Goodin 2008), their use in treatment of MPS patients for many years appears rather unlikely. Therefore, the use of natural flavonoids, which are relatively weak inhibitors of GAG synthesis with marginal, if any, adverse effects, could be one of the most promising options for SRT in MPS.

Acknowledgments This research was supported by Ministry of Sciences and Higher Education of Poland (project grant no. N N301 668540), and was operated within the Foundation for Polish Science Team Programme co-financed by the EU European Regional Development Fund (grant no. TEAM/2008-2/7). A support from Polish MPS Society is greatly acknowledged.

Conflict of interest The authors declare no conflict of interest.

Open Access This article is distributed under the terms of the Creative Commons Attribution Noncommercial License which permits any noncommercial use, distribution, and reproduction in any medium, provided the original author(s) and source are credited.

\section{References}

Arfi A, Richard M, Gandolphe C, Scherman D (2010) Storage correction in cells of patients suffering from mucopolysaccharidoses types IIIA and VII after treatment with genistein and other isoflavones. J Inherit Metab Dis 33:61-67

Beck M (2007a) Mucopolysaccharidoses: clinical features and management. In: vom Dahl S, Wendel U, Strohmeyer G (eds) Genetic metabolic disorders: management, costs and sociomedical aspects. Deutscher Arzte-Verlag, Cologne, pp 13-18

Beck M (2007b) New therapeutic options for lysosomal storage disorders: enzyme replacement, small molecules and gene therapy. Hum Genet 121:1-22 
Bulow HE, Hobert O (2006) The molecular diversity of glycosaminoglycans shapes animal development. Annu Rev Cell Dev Biol $22: 375-407$

de Azambuja E, Bedard PL, Suter T, Piccart-Gebhart M (2009) Cardiac toxicity with anti-HER-2 therapies: what have we learned so far? Target Oncol 4:77-88

Dziedzic D, Wegrzyn G, Jakobkiewicz-Banecka J (2009) Impairment of glycosaminoglycan synthesis in mucopolysaccharidosis type IIIA cells by using siRNA: a potential therapeutic approach for Sanfilippo disease. Eur J Hum Genet 18:200-205

Friso A, Tomanin R, Salvalaio M, Scarpa M (2010) Genistein reduces glycosaminoglycan levels in a mouse model of mucopolysaccharidosis type II. Br J Pharmacol 159:1082-1091

Jakobkiewicz-Banecka J, Wegrzyn A, Wegrzyn G (2007) Substrate deprivation therapy: a new hope for patients suffering from neuronopathic forms of inherited lysosomal storage diseases. J Appl Genet 48:383-388

Jakobkiewicz-Banecka J, Piotrowska E, Narajczyk M, Baranska S, Wegrzyn G (2009) Genistein-mediated inhibition of glycosaminoglycan synthesis, which corrects storage in cells of patients suffering from mucopolysaccharidoses, acts by influencing an epidermal growth factor-dependent pathway. J Biomed Sci 16:26

Kaidonis X, Liaw WC, Roberts AD, Ly M, Anson D, Byers S (2009) Gene silencing of EXTL2 and EXTL3 as a substrate deprivation therapy for heparan sulphate storing mucopolysaccharidoses. Eur J Hum Genet 18:194-199

Kato M, Takeda S, Ogawara S, Takayama S (1995) Effect of levofloxacin on glycosaminoglycan and DNA synthesis of cultured rabbit chondrocytes at concentrations inducing cartilage lesions in vivo. Antimicrob Agents Chemother 39:1979-1983

Klein CB, King AA (2007) Genistein genotoxicity: critical considerations of in vitro exposure dose. Toxicol Appl Pharmacol 224:1-11

Kwack SJ, Kim KB, Kim HS, Yoon KS, Lee BM (2009) Risk assessment of soybean-based phytoestrogens. J Toxicol Environ Health A 72:1254-1261

Li T, Perez-Soler R (2009) Skin toxicities associated with epidermal growth factor receptor inhibitors. Target Oncol 4:107-119

Li J, Richards JC (2010) Functional glycomics and glycobiology: an overview. Methods Mol Biol 600:1-8

Li N, Liu JH, Zhang J, Yu BY (2008) Comparative evaluation of cytotoxicity and antioxidative activity of 20 flavonoids. J Agric Food Chem 56:3876-3883

Malinowska M, Wilkinson FL, Bennett W et al (2009) Genistein reduces lysosomal storage in peripheral tissues of mucopolysaccharide IIIB mice. Mol Genet Metab 98:235-242

Malinowska M, Wilkinson FL, Langford-Smith KJ et al (2010) Genistein improves neuropathology and corrects behaviour in a mouse model of neurodegenerative metabolic disease. PLoS ONE 5:e14192

Matsuo M, Sasaki N, Saga K, Kaneko T (2005) Cytotoxicity of flavonoids toward cultured normal human cells. Biol Pharm Bull 28:253-259
Medina PJ, Goodin S (2008) Lapatinib: a dual inhibitor of human epidermal growth factor receptor tyrosine kinases. Clin Ther 30:1426-1447

Neufeld EF, Muenzer J (2001) The mucopolysaccharidoses. In: Scriver CR, Beaudet AL, Sly WS, Valle D (eds) The metabolic and molecular bases of inherited disease. McGraw-Hill, New York, pp 3421-3452

Piotrowska E, Jakobkiewicz-Banecka J, Baranska S et al (2006) Genistein-mediated inhibition of glycosaminoglycan synthesis as a basis for gene expression-targeted isoflavone therapy for mucopolysaccharidoses. Eur J Hum Genet 14:846-852

Piotrowska E, Jakobkiewicz-Banecka J, Tylki-Szymanska A et al (2008) Genistin-rich soy isoflavone extract in substrate reduction therapy for Sanfilippo syndrome: an open-label, pilot study in 10 pediatric patients. Curr Ther Res Clin Exp 63:166-179

Piotrowska E, Jakóbkiewicz-Banecka J, Tylki-Szymańska A, Czartoryska B, Wegrzyn A, Węgrzyn G (2009) Correlation between severity of mucopolysaccharidoses and combination of the residual enzyme activity and efficiency of glycosaminoglycan synthesis. Acta Paediatr 98:743-749

Roberts AL, Thomas BJ, Wilkinson AS, Fletcher JM, Byers S (2006) Inhibition of glycosaminoglycan synthesis using rhodamine B in a mouse model of mucopolysaccharidosis type IIIA. Pediatr Res 60:309-314

Roberts AL, Rees MH, Klebe S, Fletcher JM, Byers S (2007) Improvement in behaviour after substrate deprivation therapy with rhodamine B in a mouse model of MPS IIIA. Mol Genet Metab 92:115-121

Roberts AL, Fletcher JM, Moore L, Byers S (2010) Trans-generational exposure to low levels of rhodamine $\mathrm{B}$ does not adversely affect litter size or liver function in murine mucopolysaccharidosis type IIIA. Mol Genet Metab 101:208-213

Sanford M, Scott LJ (2009) Gefitinib: a review of its use in the treatment of locally advanced/metastatic non-small cell lung cancer. Drugs 69:2303-2328

Sherman SI (2009) Tyrosine kinase inhibitors and the thyroid. Best Pract Res Clin Endocrinol Metab 23:713-722

Takeuchi K, Ito F (2010) EGF receptor in relation to tumor development: molecular basis of responsiveness of cancer cells to EGFR-targeting tyrosine kinase inhibitors. FEBS J 277:316326

Tsai TH (2005) Concurrent measurement of unbound genistein in the blood, brain and bile of anesthetized rats using microdialysis and its pharmacokinetic application. J Chromatogr A 1073:317-322

Węgrzyn G, Jakóbkiewicz-Banecka J, Narajczyk M, Wiśniewski A, Piotrowska E, Gabig-Cimińska M, Kloska A, SłomińskaWojewódzka M, Korzon-Burakowska A, Węgrzyn A (2010) Why are behaviors of children suffering from various neuronopathic types of mucopolysaccharidoses different? Med Hypotheses 75:605-609 